\title{
Closed Loop Microfabricated Facial Reanimation Device Coupling EMG-Driven Facial Nerve Stimulation with A Chronically Implanted Multichannel Cuff Electrode
}

\author{
${ }^{1}$ Sina Askari*, ${ }^{2}$ Alessandro Presacco*,,${ }^{1,2}$ Ronald Sahyouni, ${ }^{1,2}$ Hamid Djalilian, ${ }^{1}$ Andrei Shkel, ${ }^{2}$ Harrison Lin \\ ${ }^{1}$ Department of Biomedical Engineering, ${ }^{2}$ Department of Otolaryngology, Head Neck and Surgery \\ University of California, Irvine, CA, USA \\ Email: \{askaris, presacca,rsahyoun,hdjalili, ashkel,harrison.lin \} @uci.edu \\ *These authors equally contributed to this paper
}

\begin{abstract}
Permanent facial paralysis and paresis (FP) results from damage to the facial nerve (FN), and is a debilitating condition with substantial functional and psychological consequences for the patient. Unfortunately, surgeons have few tools with which they can satisfactorily reanimate the face. Current strategies employ static (e.g., implantation of nonmuscular material in the face to aid in function/cosmesis) and dynamic options (e.g., gracilis myoneurovascular free tissue transfer) to partially restore volitional facial function and cosmesis. Here, we propose a novel neuroprosthetic approach for facial reanimation that utilizes electromyographic (EMG) input coupled to a chronically implanted multichannel cuff electrode (MCE) to restore instantaneous, volitional, and selective hemifacial movement in a feline model. To accomplish this goal, we developed a single-channel EMG-drive current source coupled with a chronically implanted MCE via a portable microprocessor board. Our results demonstrated a successful feasibility trial in which human EMG input resulted in FN stimulation with subsequent concentric contraction of discrete regions of a feline face.
\end{abstract}

\section{INTRODUCTION}

Facial nerve (FN) injury can cause permanent facial paralysis or paresis (FP), which can result in substantial clinical impairment. Deficits include dysfunctions with facial expression, proper enunciation and communication, proper blink function and corneal protection, and maintenance of oral competency. FP has an annual incidence of $11-40$ cases per 100,000 with 127,000 new cases of FP diagnosed annually in the United States alone, [1], [2]. FP can arise from FN damage due to tumors, vascular lesions (e.g. hemangioma), stroke, congenital defects, trauma, infections, or be idiopathic, with $29-35 \%$ of patients developing persistent dysfunction, including reduced facial function, spasms, and/or synkinesis, [3]-[5]. Unfortunately, surgeons have a limited arsenal of treatment options that offer patients some degree of functional restoration and cosmesis. These options range from static (e.g., implantation of nonmuscular material in the face to aid in function/cosmesis) to dynamic interventions (e.g., gracilis myoneurovascular free tissue transfer) that require staged procedures with multiple incision sites and carry a $10-15 \%$ failure rate, [6], [7].

This project was supported by grants from the American Neurotology Society, National Center for Advancing Translational Sciences (UL1TR001414), NIDCD (T32-DC010775), and MSTP (T32-GM08620).
Alternative approaches to facial reanimation may address the shortcomings of current interventions and offer treatment options to a broader range of patients who may not be eligible for or willing to undergo current treatments.

One such approach is the application of neuroprosthetic technologies to facial reanimation. In such a system, detection of movement from the normal hemiface could instantaneously activate the same muscles on the contralateral paralyzed hemiface. This approach would offer a robust, programmable, and customizable option for facial reanimation. In short, we aim to apply neuroprosthetic devices that directly stimulate cranial nerves, such as vagal nerve stimulation for epilepsy or hypoglossal nerve stimulation for obstructive sleep apnea, to the FN. Furthermore, we aim to expand the functionality of existing cuff electrode approaches by taking advantage of the ability of multichannel cuff electrodes (MCEs) to selectively stimulate motor nerve fascicles and subsequently result in selective muscle activation. Here, we present data demonstrating the feasibility of a prototype device to detect and process human electromyographic (EMG) input through a portable micro-circuit board, and deliver current to a MCE chronically implanted on a feline FN that subsequently results in concentric hemifacial contraction of discrete muscles. This neuroprosthetic strategy can be readily expanded to incorporate multichannel EMG input and current output while maintaining a small footprint, making it conducive for hermetic sealing and eventual clinical testing.

\section{METHODS}

Animals: All procedures were conducted in accordance with the NIH Animal Welfare Guidelines and with a protocol approved by the Institutional Animal Care and Use Committee at the University of California at Irvine. One female domestic shorthaired cats (Felis catus) was obtained from a research breeding colony at the University of California at Davis. The cat was recorded for seven months post-electrode implantation.

Anesthesia: A light level of anesthesia was induced with an intramuscular injection of $.7 \mathrm{~mL}$ of ketamine $(20 \mathrm{mg} / \mathrm{kg})$ and $.3 \mathrm{~mL}$ of acepromazine $(1 \mathrm{mg} / \mathrm{kg})$; with a booster dose of $.3 \mathrm{~mL}$ of ketamine given when necessary to maintain stable sedation. 
MCE Specifications: A custom 8-channel MCE (MicroProbes for Life Science, Gaithersburg, MD, USA) with an inner diameter of $1.5 \mathrm{~mm}$ was used. The MCE contained two "rings" of electrodes with each ring containing 4 individual 100 micrometer rectangular (tripolar) platinum contacts housed within a silicon enclosure. Each electrode was positioned at $0^{\circ}, 90^{\circ}, 180^{\circ}$ and $270^{\circ}$ around the ring, and perpindicular to the neural epineurium (Fig. 1). This configuration allowed for monopolar stimulation of four discrete neural locations within each ring, which ultimately allowed for eight individual contact points with the nerve. Field steering allowed for stimulation of 2 (or more) contacts simultaneously.

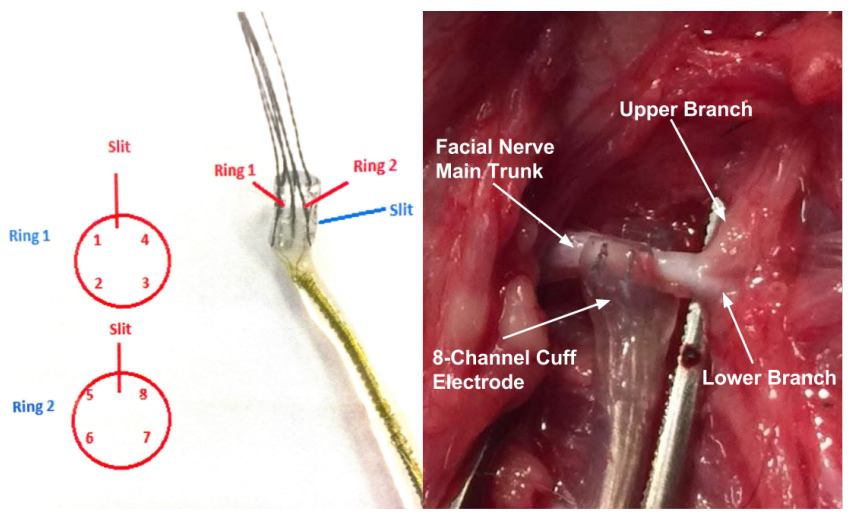

Fig. 1. (Left) 8-channel MCE with 2 electrode "rings", each with 4 rectangular platinum electrodes in a silicon enclosure $90^{\circ}$ apart. (Right) Intraoperative image of implanted MCE.

Surgical Implantation: A pre-auricular incision was made under sterile conditions and dissection through the parotid gland proceeded until the FN trunk was identified and skeletonized. The MCE was positioned at the main trunk of the FN, and fastened shut with incorporated nylon sutures. The positioning (i.e., site and angle of contact) was not predetermined; positioning of the MCE was dictated by the gross-surgical anatomy and FN access. The MCEs wire connectors were tunneled and fastened to a skull-mounted Omnetics (Minneapolis, MN, U.S.A.) connector that was encapsulated by a stainless-steel cylinder. Acrylic sealant was used at the floor of the cylinder and the base of the connector to make sure the cylinder interior was inaccessible from exposed tissue junctions. The muscle and skin layers were sutured and a screw-on cap was placed over the stainlesssteel cylinder.

Stimulus Generation: Two different set-ups were used to generate biphasic electrical pulses ( $82 \mu s$ duration/phase).

In the first set-up (TDTCS), electrical pulses were generated by a custom optically isolated 16-channel current sources controlled by 16-bit digital-to-analog converters (TDT RX8). Each channel had a maximum output of 1 $\mathrm{mA}$ and four different current intensity were tested: $\sim 1$, $\sim 0.56$ and $\sim 0.36 \mathrm{~mA}$. In order to better investigate EMG responses, in this phase of our study we presented only a single biphasic pulse rather than train of pulses. This allowed us to have artifact-free EMG, as the stimulus artifact was

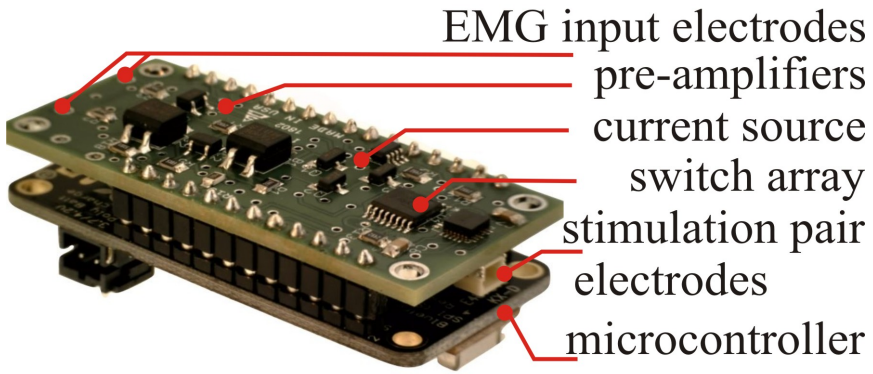

Fig. 2. The developed EMG-driven nerve stimulation prototype. A custom daughter board was developed to interface with the commercially available Adafruit Feather $32 \mathrm{u} 4$ microcontroller.

confined to the first $1 \mathrm{~ms}$ of data.

In the second set-up (Portable Platform), a miniaturized EMG-driven current source was developed (Fig. 2-3). This platform includes an EMG pre-amplifier, analog EMG signal conditioning, an 8-bits microcontroller, buffer electronic circuitry, current source, and digital switches to generate a biphasic current stimulus and with an onboard Li-ion battery for power management. The platform is capable of mapping EMG data to biphasic electrical current pulses in response to detection of EMG activity. Raw EMG activity from volitional human muscle activity was detected and recorded by three surface skin electrodes (one active and one reference electrode were used for recording the signal and the third was used as a ground electrode), and enhanced by an EMG preamplifier (INA333). The EMG activity was high-pass filtered to eliminate DC voltage (cut-off frequency $=1 \mathrm{~Hz}$ ) and was connected to the 10-bit Analog-to-Digital converter

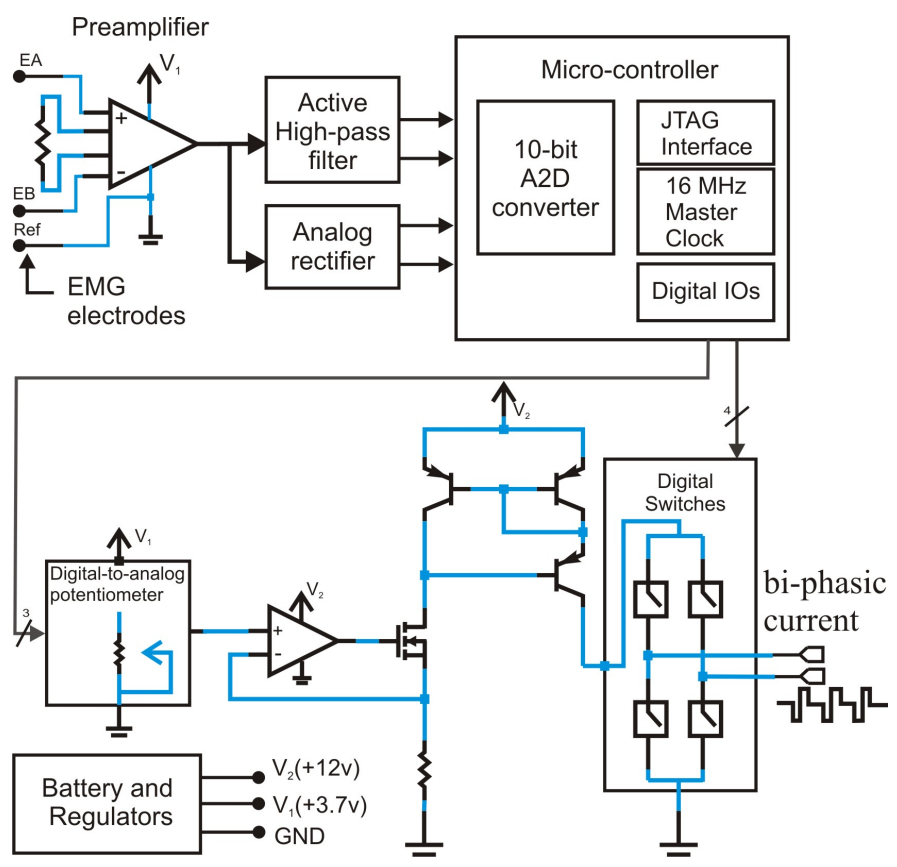

Fig. 3. Detailed schematic of the EMG-driven biphasic current stimulator. Input EMG data are processed and translated to output-current stimulus amplitude which ultimately activated feline facial muscles. 
of the ATmega32U4 microcontroller. The microcontroller was programmed to read and process EMG input data every $16 \mu s$ and to control the current source and the frequency of the digitally controlled switch arrays (ADG611). The platform, therefore, becomes a closed loop EMG feedback controller which is capable of delivering biphasic current pulses when EMG activity surpasses a certain threshold. For this study, the maximum current level up to $\sim 1.33 \mathrm{~mA}$ was utilized. Circuit power consumption including the microcontroller at full operation under EMG activity was measured to be $330 \mathrm{~mW}$. For the purpose of this study, which was to compare EMG recorded between the developed Portable Platform and TDTCS, we limited the stimulation to a single biphasic pulse, even though the platform has the flexibility to generate a train of pulses to any rate, the only limitation being the ADC frequency conversion.

Electromyography (EMG): Following biphasic current delivery to a MCE channel, EMG activity from concentric muscle contraction in the feline hemiface was detected and recorded using TDT hardware with clinical sub-dermal electrodes. Four active electrodes were used to record from four different facial muscles: levator, orbicularis oris, orbicularis oculi and nasalis. The reference and the ground were placed in the lateral/long triceps head on the front limb of the cat. The sampling frequency was $24,414 \mathrm{~Hz}$. The TDTCS set-up included a high-pass digital filter with a cut-off frequency at $10 \mathrm{~Hz}$, while the prototype set-up included a high-pass digital filter with a cut-off frequency at $1 \mathrm{~Hz}$. The signal was downsampled in real-time to $3,052 \mathrm{~Hz}$ and then band-pass filtered offline using a 4th order Butterworth filter $(100-1500 \mathrm{~Hz})$.

\section{RESULTS}

TDTCS set-up: Figure 4, shows the results when using the TDTCS set-up and stimulating facial muscles through channel 1 of the MCE. The first $1 \mathrm{~ms}$ of the waveform represents stimulus artifact elicited by the biphasic electrical pulse. Levator and nasalis were activated when applying a current of $1 \mathrm{~mA}$ and resulting EMG activity was observed at around $5 \mathrm{~ms}$ following stimulus onset. Conversely, no EMG was detected from oris and oculi. As the intensity of the current was decreased down to $0.56 \mathrm{~mA}$, excellent muscle selectivity was achieved. At this current level, EMG activity from only the levator muscle was recorded, while the other three muscles remained "silent". The current intensity of 0.3 $\mathrm{mA}$ was not sufficient to stimulate any of the four muscles and only stimulus artifact was recorded.

Prototype set-up vs TDTCS set-up: Figure 5, shows the EMG responses recorded from a representative muscle (nasalis) elicited by current delivered by the TDTCS setup (panel A) and the developed prototype (panel B). The waveform generated from the prototype demonstrates good agreement with the waveform triggered by the TDTCS setup, in terms of both the strength of the response (similar amplitude values) and the latency $(\sim 5 \mathrm{~ms})$ of the most prominent positive peak post stimulus onset.
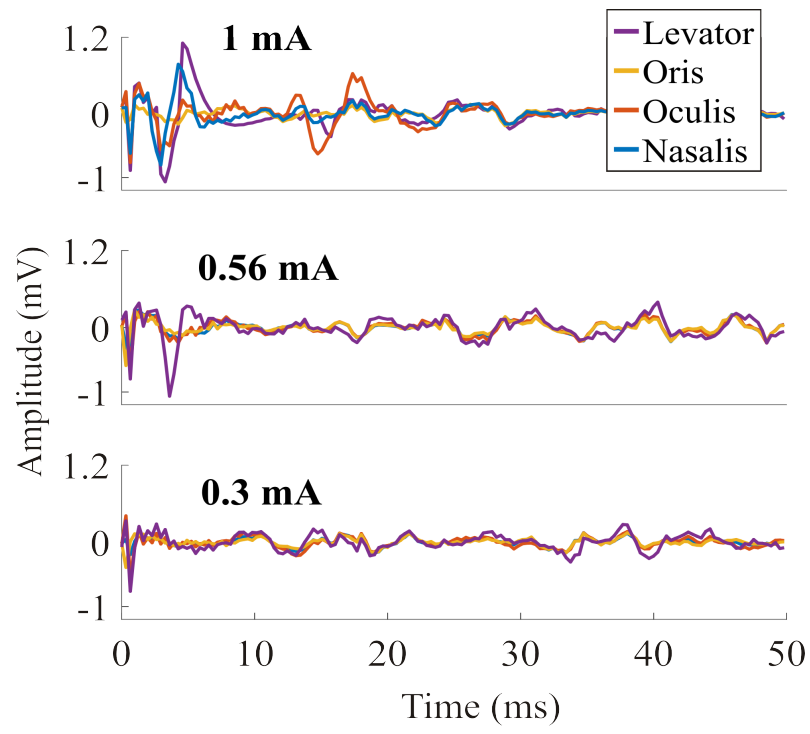

Fig. 4. EMG recorded simultaneously from four muscles: levator, oris, oculi and nasalis. Channel 1 of the MCE was used to deliver current. Each raw signal represents a different level of current presented. The best selectivity was achieved with $0.56 \mathrm{~mA}$, where only levator was activated.

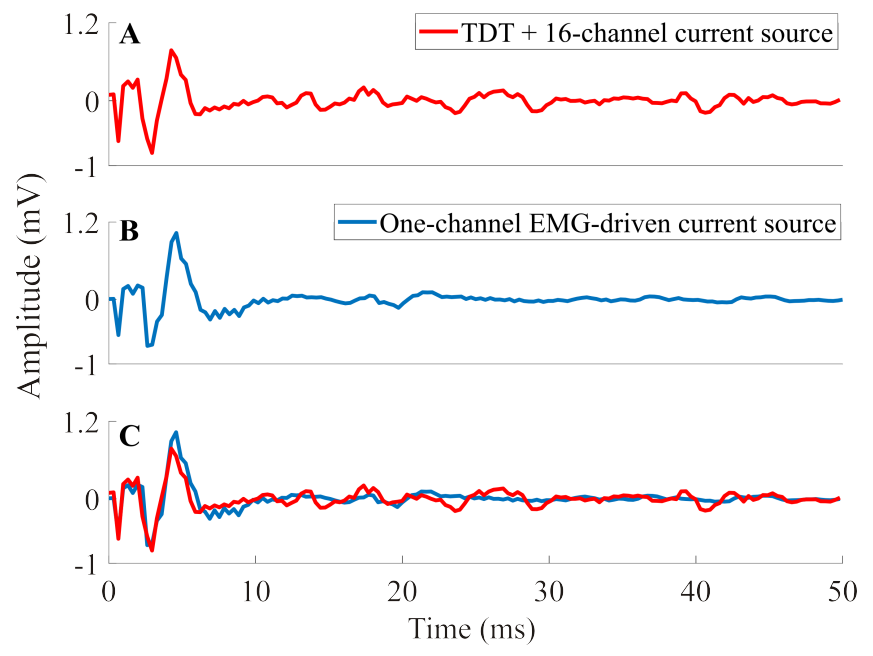

Fig. 5. EMG responses recorded at month 7 from nasalis elicited by the TDTCS set-up (panel A) and by the developed prototype (panel B). The two responses are comparable (Panel C) both in terms of signal strength (similar amplitude) and latency $(\sim 5 \mathrm{~ms})$.

\section{DISCUSSION}

In this paper, we presented a novel application of a neuroprosthetic device to provide a closed loop facial reanimation system with direct translational implications for FP. One cat was implanted with MCE electrodes and was tested with the TDTCS set-up every two weeks starting from one month post implantation up to six months. After six months, EMG was recorded every month and the data presented in this paper refer to recording obtained seven months postimplantation from channel 1 . Following 7 months of chronic MCE implantation, the MCE remained functional, and able to activate discrete facial muscles in a feline hemiface. This study elucidated stimulation parameters required to trigger 
muscle activation, and the data suggest that MCEs have the ability to selectively stimulate individual muscles, as long as the current level does not saturate the nerve and depolarize all neural fibers. This is due to the fact that delivering suprathreshold levels of current to a motor nerve can saturate the fascicular selectivity and generate global depolarization of all motor nerve fibers. In this study, we observed that a current intensity of $0.56 \mathrm{~mA}$ would be ideal to activate the levator, while keeping the other three muscles we recorded from "silent". As we increased the current level up to $1 \mathrm{~mA}$, this selectivity is partially lost and EMG from the nasalis is also detected. The activation of two muscles at $1 \mathrm{~mA}$ is likely due to the spread of excitation elicited by the increase in current being delivered.

Coupling the implanted MCE to the prototype device, which utilized volitional EMG input to deliver graded biphasic current pulses to specific MCE channels, demonstrated a successful feasibility study that is the first step towards developing an implantable clinical device. We purposely used a suprathreshold level of current comparable to the one tested with the TDTCS set-up that would allow us to elicit muscle response. Both amplitude and latency of the most prominent positive peak recorded post-stimulus onset were comparable with the one generated using the TDTCS set-up.

Ultimately, the expansion of the developed prototype technology to incorporate multichannel input and output functionality would allow for the detection of EMG activity from multiple facial muscles of a normal hemiface, which would then drive current delivery to the appropriate channels of the implanted MCE that would subsequently activate the same muscles on the contralateral paralyzed hemiface. The ability for this system to function requires the presence of neural fibers, which have the potential to regenerate following injury, but may be too weak to result in concentric muscle contraction due to insufficient endogenous acetylcholine release at the neuromuscular junction (NMJ), [8], [9]. In this case, the application of exogenous electrical current could potentially amplify endogenous signals and provide tone to the paralyzed hemiface, as well as selectively stimulate individual facial muscles to provide volitional, graded, and instantaneous bilateral facial movement. Furthermore, exogenous electrical stimulation has been demonstrated to facilitate endogenous peripheral nerve regeneration and upregulate NMJ formation, which can assist with FP recovery and strengthen endogenous facial function, [10]-[12] .

For the purpose of this study, we limited the current level of the pulses to $1.33 \mathrm{~mA}$. However, the developed prototype has been designed to be able to generate a wide range of current levels, that are regulated by an 8-bit digital potentiometer. The ultimate goal of the prototype will be to generate graded levels of current in accordance with the amplitude of the EMG. This would ideally allow FP patients to have different levels of activation of the paralyzed facial muscle, thus allowing them to mimic the strength of the healthy facial muscle being used to drive the current source.

Neuroprosthetic technologies have been readily used in a variety of clinical settings with great success. Cuff electrodes, specifically, have been successfully employed to treat obstructive sleep apnea (hypoglossal nerve stimulation), epilepsy and heart failure (vagus nerve stimulation), and are even being tested in the neuromuscular system of the extremities to treat paralysis. [13]-[15] We hope to encourage further investigation into the utilization of closed loop implantable devices that can selectively depolarize discrete neural fascicles, and subsequently activate individual muscles. Possible inputs could vary from EMG (as in the present study), to electroencephalogram data, which can then be used to drive a variety of current output parameters, with wide ranging opportunities for clinical translatability.

\section{REFERENCES}

[1] J. A. McCaul, L. Cascarini, D. Godden, D. Coombes, P. A. Brennan, and C. J. Kerawala, "Evidence based management of Bell's palsy," British Journal of Oral and Maxillofacial Surgery, vol. 52, no. 5, pp. 387-391, 2014.

[2] J. N. Bleicher, S. Hamiel, J. S. Gengler, and J. Antimarino, "A survey of facial paralysis: etiology and incidence." Ear, nose, \& throat journal, vol. 75, no. 6, pp. 355-358, 1996.

[3] M. May, R. Wette, W. B. Hardin, and J. Sullivan, "The use of steroids in Bell's palsy: a prospective controlled study," The Laryngoscope, vol. 86, no. 8, pp. 1111-1122, 1976.

[4] M. May, S. R. Klein, and F. H. Taylor, "Idiopathic (Bell's) facial palsy: natural history defies steroid or surgical treatment." The Laryngoscope, vol. 95, no. 4, pp. 406-409, 1985.

[5] E. Peitersen, "Bell's palsy: the spontaneous course of 2,500 peripheral facial nerve palsies of different etiologies," Acta Oto-Laryngologica, vol. 122, no. 7, pp. 4-30, 2002.

[6] P. K. Bhama, J. S. Weinberg, R. W. Lindsay, M. H. Hohman, M. L. Cheney, and T. A. Hadlock, "Objective outcomes analysis following microvascular gracilis transfer for facial reanimation: a review of 10 years experience," JAMA facial plastic surgery, vol. 16, no. 2, pp. 85-92, 2014.

[7] J. E. Adams, M. F. Kircher, R. J. Spinner, M. E. Torchia, A. T. Bishop, and A. Y. Shin, "Complications and outcomes of functional free gracilis transfer in brachial plexus palsy," Acta Orthop Belg, vol. 75, no. 1, pp. 8-13, 2009.

[8] G. Stoll and H. W. Müller, "Nerve injury, axonal degeneration and neural regeneration: basic insights," Brain pathology, vol. 9, no. 2, pp. 313-325, 1999.

[9] X. Navarro, M. Vivó, and A. Valero-Cabré, "Neural plasticity after peripheral nerve injury and regeneration," Progress in neurobiology, vol. 82, no. 4, pp. 163-201, 2007.

[10] A. Eberstein and B. R. Pachter, "The effect of electrical stimulation on reinnervation of rat muscle: contractile properties and endplate morphometry," Brain research, vol. 384, no. 2, pp. 304-310, 1986.

[11] A. M. Stanco and M. J. Werle, "Agrin and acetylcholine receptor distribution following electrical stimulation," Muscle \& nerve, vol. 21, no. 3, pp. 407-409, 1998.

[12] T. Fukazawa, M. Matsumoto, T. Imura, E. Khalesi, T. Kajiume, Y. Kawahara, K. Tanimoto, and L. Yuge, "Electrical stimulation accelerates neuromuscular junction formation through ADAM19/neuregulin/ErbB signaling in vitro," Neuroscience letters, vol. 545, pp. 29-34, 2013.

[13] R. H. Howland, "Vagus nerve stimulation," Current behavioral neuroscience reports, vol. 1, no. 2, pp. 64-73, 2014.

[14] P. R. Eastwood, M. Barnes, J. H. Walsh, K. J. Maddison, G. Hee, A. R. Schwartz, P. L. Smith, A. Malhotra, R. D. McEvoy, J. R. Wheatley et al., "Treating obstructive sleep apnea with hypoglossal nerve stimulation," Sleep, vol. 34, no. 11, pp. 1479-1486, 2011.

[15] K. H. Polasek, H. A. Hoyen, M. W. Keith, R. F. Kirsch, and D. J. Tyler, "Stimulation stability and selectivity of chronically implanted multicontact nerve cuff electrodes in the human upper extremity," IEEE Transactions on Neural Systems and Rehabilitation Engineering, vol. 17, no. 5, pp. 428-437, 2009. 